\title{
Carga de la enfermedad: insuficiencia renal, diálisis-hemodiálisis y trasplante renal en México. Costo de la enfermedad
}

\author{
Burden of disease: end stage renal disease, dialysis-hemodialysis \\ and kidney transplantation in Mexico
}

\author{
Aczel Sánchez-Cedillo, * José Cruz-Santiago, ${ }^{\ddagger}$ Fabiola Beatriz Mariño-Rojas, ${ }^{\S}$ \\ Sergio Hernández-Estrada," Catalina García-Ramírez" \\ * Coordinación Institucional de Trasplantes, Instituto de Seguridad y Servicios Sociales de los Trabajadores del Estado (ISSSTE). \\ ‡ Unidad de Trasplantes, Hospital de Especialidades, Centro Médico Nacional «La Raza», Instituto Mexicano \\ del Seguro Social (IMSS). Ciudad de México, México. \\ $\S$ Medical Manager PIH-Rare Diseases, Pfizer México. \\ " Centro Médico Nacional «20 de Noviembre», Instituto de Seguridad y Servicios Sociales \\ de los Trabajadores del Estado (ISSSTE). Ciudad de México, México. \\ " Hospital de Especialidades, Centro Médico Nacional «La Raza», Instituto Mexicano del Seguro Social (IMSS). Ciudad de México, México.
}

\section{RESUMEN}

Introducción: La enfermedad renal crónica (ERC) es una enfermedad multifactorial de carácter progresivo e irreversible. En esta enfermedad la función renal se encuentra deteriorada; en estadios avanzados requiere, por lo general, terapias de reemplazo renal (TRR). El elevado costo del tratamiento sustitutivo de la ERC representará un importante reto para los sistemas de salud. En nuestro país, históricamente ha predominado el uso de diálisis peritoneal, aunque recientemente se ha dado impulso a la hemodiálisis. Por un lado, en México, la hemodiálisis sigue siendo poco accesible para la mayoría de los pacientes. Por otro lado, el trasplante renal (TR) es la única modalidad de TRR que realmente previene el desarrollo

\begin{abstract}
Introduction: Chronic Kidney Disease (CKD) is a multifactorial disease of a progressive and irreversible nature, in which renal function is impaired that in end stages requires renal replacement therapies (RRT). The high cost of CKD replacement therapy will represent a major challenge for health systems. Mexico is a country in which the use of peritoneal dialysis has historically predominated, although hemodialysis has recently been encouraged. On the other hand, in Mexico, hemodialysis remains poorly accessible for most patients. Kidney transplantation (KT) is the only modality of RRT that really prevents the development of uremia, but, unfortunately, not all patients with CKD are candidates for KT. For this
\end{abstract}

\footnotetext{
Abreviaturas:

CENATRA = Centro Nacional de Trasplantes.

$\mathrm{DM}=$ diabetes mellitus.

DPA = diálisis peritoneal automatizada.

DPCA = diálisis peritoneal continua ambulatoria.

$\mathrm{ECD}=$ enfermedades crónico-degenerativas.

$\mathrm{ERC}=$ enfermedad renal crónica.

$\mathrm{ERCT}=$ enfermedad renal crónica terminal.

GRD = Grupo Relacionado por el Diagnóstico.
}

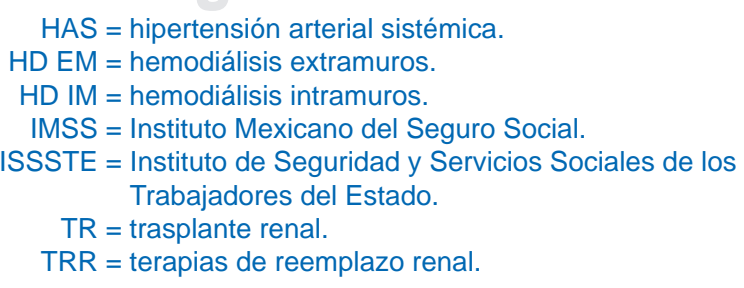


de uremia, pero desgraciadamente no todos los pacientes con ERC son candidatos a TR. Por esta razón, es necesaria una evaluación exhaustiva de los candidatos para TR con el fin de maximizar los recursos económicos y humanos. Objetivo: Determinar cuál es la alternativa de menor costo de atención en pacientes con diálisis-hemodiálisis en comparación con los pacientes en TR desde la perspectiva de dos instituciones públicas como son el Instituto Mexicano del Seguro Social (IMSS) y el Instituto de Seguridad y Servicios Sociales de los Trabajadores del Estado (ISSSTE). Material y métodos: Estudio retrospectivo para estimar los costos promedio de atención de la diálisis-hemodiálisis y del TR. Los costos promedio estimados en salud fueron evaluados desde el punto de vista de los prestadores de servicios públicos de salud, en este caso, el IMSS y el ISSSTE. La frecuencia de recursos utilizados de los pacientes con diálisis-hemodiálisis y TR se estimó a través de la propia información de la experiencia de atención de los médicos tratantes de cada institución durante 2017. Sólo se consideraron los costos médicos directos de los pacientes (insumos y/o recursos que utilizaron en un año), con un seguimiento posterior al año tres y seis del seguimiento. Resultados: El costo de atención anual promedio per cápita para el primer año de la diálisis-hemodiálisis fue de 465,485.96 y 783,780.44 pesos, respectivamente, siendo el costo por las complicaciones en el caso de la diálisis el de mayor tamaño, lo cual representa $54.6 \%$ del costo total de atención; en tanto que en los pacientes con hemodiálisis las sesiones de tratamiento representaron $50.8 \%$ del costo total. El costo de atención anual promedio de los pacientes con TR per cápita para el año uno fue de $\$ 862,490.51$, en el que el costo por las complicaciones fue el que generó el mayor gasto de atención, lo cual representa $52.6 \%$ del costo total de atención. Al realizar el seguimiento (tres y seis años) se observó una disminución en los costos de los pacientes con TR, con un decremento en el costo frente a diálisis de 44 y $43 \%$, así como de 36 y $39 \%$ frente a los pacientes con hemodiálisis, respectivamente. Conclusiones: El TR resultó ser la alternativa de menor costo para el manejo de estos pacientes, siendo la hemodiálisis la terapia de mayor costo. Desde una perspectiva económica, el TR debería ser la intervención de mayor promoción y aplicación en cuanto a las tres TRR.

Palabras clave: Costo de la enfermedad, insuficiencia renal, diálisis, hemodiálisis, trasplante renal, seguridad social.

\section{INTRODUCCIÓN}

En México, el envejecimiento de la población y la adopción de estilos de vida no saludables conllevan un incremento en la incidencia de enfermedades crónico-degenerativas (ECD). Esto ha traído una serie de nuevos retos, entre los que destacan la demanda creciente en los servicios médicos y el impacto económico significativo para el sistema de salud. En este sentido, la enfermedad renal crónica $(E R C)$ es el resultado causado por diversas ECD, entre las que destacan la diabetes mellitus (DM) y la reason, a thorough evaluation of the candidates for $K T$ is necessary in order to maximize economic and human resources. Objective: To determine the alternative of lower cost of care in patients with Dialysis-Hemodialysis compared to patients in KT from the perspective of two public institutions such as the Mexican Social Security Institute (IMSS) and the Institute of Social Security and Services of State Workers (ISSSTE). Material and methods: Retrospective study, to estimate the average costs of Dialysis-Hemodialysis and KT care. The estimated average costs in health were evaluated from the point of view of public health service providers, in this case the IMSS and ISSSTE. The frequency of use of resources of patients with dialysis-hemodialysis and KT was estimated through the information of the care experience of the treating physicians of each Institution during 2017. Only the direct medical costs of the patients were considered (inputs and/or resources used in one year), with a follow-up after year three and six of followup. Results: The average annual cost of care per capita for the first year of dialysis-hemodialysis was $\$ 465,485.96$ and $\$$ $783,780.44$ respectively, the cost for complications in the case of Dialysis being the heaviest representing $54.6 \%$ of the total cost of care while in patients with hemodialysis, treatment sessions accounted for $50.8 \%$ of the total cost. The average annual cost of care for patients with KT per capita for year 1 was $\$ 862,490.51$, where the cost for complications was the one that generated the greatest care expense, representing $52.6 \%$ of the total cost of care. When monitoring (year three and six), there is a decrease in the costs of patients with KT, with a decrease in the cost against Dialysis of 44 and $43 \%$, as well as 36 and $39 \%$ compared to patients in hemodialysis respectively. Conclusions: $K T$ proved to be the lowest cost alternative for the management of these patients, being hemodialysis the most expensive therapy. From an economic perspective, the KR should be the intervention with the greatest promotion and application regarding the three $R R T$.

Keywords: Burden of disease, dialysis, hemodialysis, end stage renal disease, kidney transplantation, social security.

hipertensión arterial sistémica (HAS), fenómeno que ocurre de manera similar en todo el mundo. La ERC es un proceso multifactorial de carácter progresivo e irreversible que, frecuentemente, conduce a un estado terminal en el cual la función renal se encuentra lo suficientemente deteriorada como para ocasionar la muerte del paciente, o bien para requerir terapias de reemplazo renal (TRR). ${ }^{1}$

De igual forma, en nuestro país, se ha observado un incremento importante en la prevalencia e incidencia de ERC. En la actualidad, esta enfermedad se considera una pandemia que afecta aproximadamente a 
Probabilidad de supervivencia

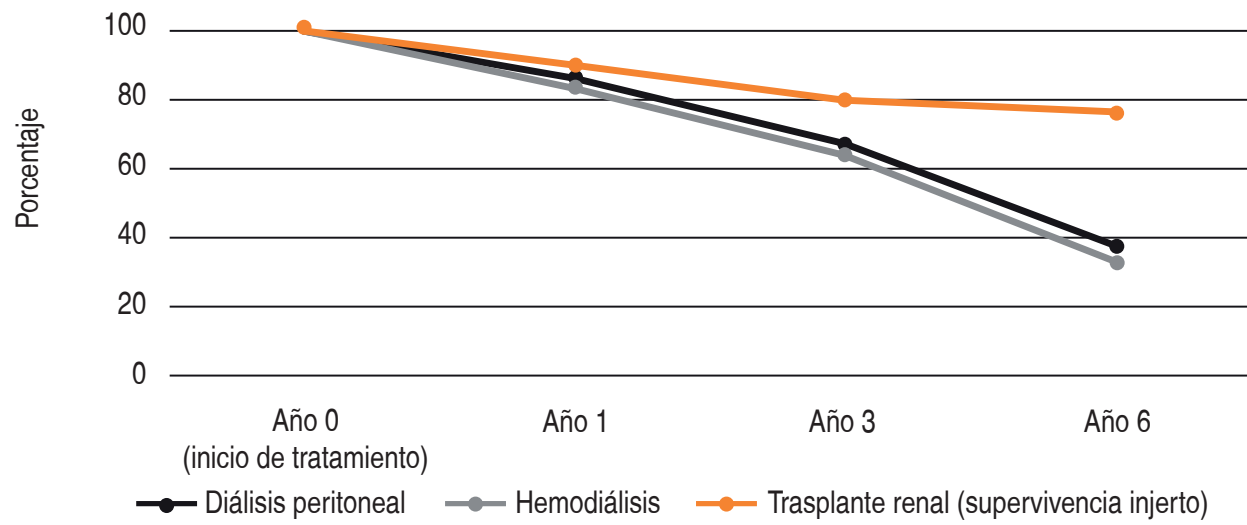

Figura 1:

Probabilidad de supervivencia para diálisis peritoneal continua ambulatoria, hemodiálisis y trasplante renal. ${ }^{7}$
$10 \%$ del total de la población adulta de todas partes del mundo. ${ }^{2}$ Cabe señalar que México carece de un registro de pacientes con ERC, por lo que se desconoce cuál es el número de pacientes (en cualquiera de sus estadios), los grupos de edad y género más afectados, así como el comportamiento propio de los programas; aun así, para nuestro país, se estima una incidencia y una prevalencia de 377 y 1,142 casos por millón de habitantes, respectivamente. ${ }^{3}$

Las causas de ERC se pueden agrupar en enfermedades vasculares, glomerulares, del túbulo intersticial y uropatías obstructivas. Actualmente, la etiología más frecuente en la República Mexicana es la secundaria a DM, que es responsable hasta de $50 \%$ de los casos de ERC, seguida en frecuencia por la HAS y las glomerulonefritis. La evolución de la ERC es variable, aunque depende de su etiología; por lo general, comienza de manera insidiosa y progresa lentamente con los años. La etapa 5 de la ERC, conocida como enfermedad renal crónica terminal (ERCT), ocurre cuando es necesario recurrir a una TRR; sin embargo, no todos los pacientes progresan hasta la etapa final: datos recientes han mostrado que los pacientes con ERC tienen de cinco a 10 veces más probabilidades de morir antes de alcanzar la etapa terminal. ${ }^{4}$

La ERCT constituye un problema de salud pública asociado con una elevada morbimortalidad, grandes costos y una calidad de vida disminuida. En nuestro país, el problema de la ERCT posee dimensiones alarmantes y, con base en proyecciones, se estima que el número de casos continuará en aumento; de hecho, si las condiciones actuales persisten, se prevé que para el año 2025 habrá cerca de 212 mil casos y se registrarán casi 160 mil muertes relacionadas con dicha enfermedad. ${ }^{1}$
Con esta información también es necesario considerar el elevado costo del tratamiento de la ERCT, el cual representará un importante reto para los sistemas de salud, en especial para los países en desarrollo. ${ }^{5}$ En este contexto, se vuelve relevante el uso de la TRR para preservar la vida del paciente, de ahí que estas terapias sean llamadas "soporte de vida». La TRR incluye la diálisis peritoneal, la hemodiálisis y el trasplante renal (TR). ${ }^{1}$ En México, de manera histórica ha predominado el uso de diálisis peritoneal, aunque recientemente se ha dado impulso a la hemodiálisis. Este tratamiento de sustitución ha tenido notables avances tecnológicos, por lo que, durante las últimas dos décadas, su costo ha disminuido de manera muy importante hasta ubicarse en un nivel competitivo en relación con la diálisis peritoneal. Sin embargo, la hemodiálisis sigue siendo poco accesible para la mayoría de los pacientes. ${ }^{4}$

De acuerdo con la información por parte del Instituto Mexicano del Seguro Social (IMSS), institución que atiende a cerca de $73 \%$ de la población mexicana que requiere diálisis o trasplante, para el año 2014 el tratamiento de la ERCT representó para esta institución el $15 \%$ del gasto total anual de su mayor programa (Seguro de Enfermedades y Maternidad), esto es aproximadamente 13,250 millones de pesos; esto significa que dicho gasto se invirtió en tan sólo $0.8 \%$ de los derechohabientes (población con ERCT). Además, se estima que si en nuestro país el acceso a diálisis fuese universal se requeriría una inversión de más de 33,000 millones de pesos anuales, lo que representaría cerca de $40 \%$ del presupuesto nacional destinado al sector salud. ${ }^{6}$

En cuanto al TR, en la actualidad se considera como la mejor opción de tratamiento para la ERC; 
no obstante, en nuestro país, ésta no es una solución viable debido a la falta de donaciones, los altos costos iniciales y el nivel de deterioro orgánico que presentan los pacientes por las enfermedades primarias. ${ }^{1}$ Según cifras del informe anual del Centro Nacional de Trasplantes (CENATRA), para 2017 en México se tenía un total de 13,634 pacientes receptores en espera de un riñón y, durante ese mismo año, se realizaron un total de 3,150 trasplantes renales. ${ }^{8}$ En tanto que para el primer semestre de 2018 (corte al 03 de julio de 2018), se tenía en espera de un TR a 14,258 receptores y se habían realizado un total de 1,523 trasplantes renales. ${ }^{9}$

Ahora bien, se considera que el TR es la única modalidad de TRR que realmente previene el desarrollo de uremia, pero, por desgracia, no todos los pacientes con ERC son candidatos a TR; además, la evaluación de los candidatos a TR minimiza la morbimortalidad, al igual que mejora la calidad de vida. Hay varios estudios que demuestran una supervivencia mayor en los pacientes con TR que en pacientes con diálisis, ${ }^{10}$ al igual que existen varios estudios que documentan una mayor supervivencia de los pacientes trasplantados en los que previamente no utilizaron diálisis. ${ }^{11}$ También se ha observado que los mejores resultados se obtienen en pacientes que son trasplantados de forma temprana. Por esto, es necesaria una evaluación exhaustiva de los candidatos para TR con el fin de maximizar los recursos económicos y humanos.

Como se mencionó, la ERC constituye un reto económico para los sistemas de salud, pues representa una de las enfermedades más costosas a nivel mundial en materia de tratamiento y su costo continúa aumentando. Por ello, la utilización de las modalidades de TRR varía en los diversos países. En México, de acuerdo con cifras reportadas del IMSS a través de 212 hospitales generales y regionales, así como de 13 Unidades Médicas de Alta Especialidad distribuidos en el territorio nacional, durante 2014 la distribución de los pacientes por modalidad dialítica fue cerca de $59.0 \%$ para pacientes con diálisis (32.0\% para pacientes con diálisis peritoneal continua ambulatoria [DPCA] y $27.0 \%$ para pacientes con diálisis peritoneal automatizada [DPA]) y de $41.0 \%$ para pacientes en hemodiálisis (17.0\% para pacientes con hemodiálisis intramuros [HD IM] y $24.0 \%$ para pacientes con hemodiálisis extramuros [HD EM]). Durante este periodo de tiempo, el IMSS reportó que la HD EM fue la de mayor costo promedio, siendo aproximadamente el costo anual de 234,949 pesos para cada paciente, seguida de la DPA y de la HD IM, con un costo anual por paciente de 72,983 y de 61,482 pesos, respectivamente. Por último, la DPCA resultó ser la TRR de menor costo anual con 48,672 pesos por paciente..$^{12,13}$

Con base en estos resultados y con la situación actual de nuestro sistema de salud, los retos de la salud pública actual que afronta desde el marco de reformas se encuentran influidos por dos aspectos que no pueden dejarse de lado: las necesidades infinitas de la población y la escasez de recursos financieros. De ahí, surge la necesidad de generar insumos que permitan tomar decisiones cuya implementación permita disminuir, en la medida de lo posible, los efectos de la falta de equidad, eficiencia y calidad en la prestación de servicios médicos. De esta manera, la importancia de la evaluación de los costos y la efectividad de intervenciones radica en su potencial uso costo-efectivo de los recursos dirigidos a los problemas prioritarios de salud. Si bien es cierto que existen estudios sobre el costoefectividad para la ERCT en países de ingreso alto, cuyos resultados se orientan hacia la promoción del $\mathrm{TR}$, sin entrar en mayor detalle, también es cierto que en países de ingreso medio como México, este tipo de estudios escasamente han sido desarrolla-

Tabla 1: Insumos utilizados por año en diálisis peritoneal.

\begin{tabular}{lccc}
\hline & \multicolumn{3}{c}{ Núm. de insumos } \\
\cline { 2 - 4 } Frecuencia de insumos utilizados & Año 1 & Año 3 & Año 6 \\
\hline Consultas totales (control y seguimiento) [Rango] & $8[4-12]$ & $8[4-12]$ & $11[4-18]$ \\
Estudios de laboratorio [Rango] & $4[4-4]$ & $4[4-4]$ & $4[4-4]$ \\
Estudios de gabinete (radiodiagnósticos y electrodiagnóstico) [Rango] & $3[2-4]$ & $3[2-4]$ & $2.5[2-3]$ \\
Insumos para la diálisis [Rango] & $12[12-12]$ & $12[12-12]$ & $12[12-12]$ \\
Costo de la cirugía (catéter) [Rango] & $1[1-1]$ & $1[1-1]$ & $0[0-0]$ \\
\hline
\end{tabular}


Tabla 2: Insumos utilizados por año en hemodiálisis.

\begin{tabular}{lccr}
\hline & \multicolumn{3}{c}{ Núm. de insumos } \\
\cline { 2 - 4 } Frecuencia de insumos utilizados & Año 1 & Año 3 & Año 6 \\
\hline Consultas totales (control y seguimiento) [Rango] & $8[4-12]$ & $8[4-12]$ & $8[4-12]$ \\
Estudios de laboratorio [Rango] & $7[4-10]$ & $7[4-10]$ & $7[4-10]$ \\
Estudios de gabinete (radiodiagnósticos y electrodiagnósticos) [Rango] & $2[2-2]$ & $2[2-2]$ & $2[2-2]$ \\
Procedimiento de hemodiálisis (sesión de hemodiálisis) [Rango] & $144[144-144]$ & $144[144-144]$ & $144[144-144]$ \\
Cirugía para fístula arteriovenosa [Rango] & $1[1-1]$ & $1[1-1]$ & $0[0-0]$ \\
Cirugía para implantación de catéter [Rango] & $1[1-1]$ & $1[1-1]$ & $0[0-0]$ \\
\hline
\end{tabular}

dos, y por lo tanto, los programas de atención se organizan a partir de los resultados de otros países. Así, hoy en día, se trata de retomar metodologías de análisis desarrolladas en países de ingreso alto y aplicarlas a las condiciones y sistemas de salud de países con ingreso medio, como lo es en nuestra nación. Por lo tanto, la realización de este tipo de estudios es prioridad en nuestro sistema de salud, pues con ello se podrán tomar las mejores decisiones dentro del medio y elegir la terapia que sea más costo-efectiva tanto para el paciente como para las instituciones de salud. ${ }^{14,15}$

\section{MATERIAL Y MÉTODOS}

Desde la perspectiva de dos instituciones públicas como son el Instituto Mexicano del Seguro Social (IMSS) y el Instituto de Seguridad y Servicios Sociales de los Trabajadores del Estado (ISSSTE), se realizó un estudio retrospectivo que busca estimar los costos promedio de la atención de pacientes en diálisis-hemodiálisis en comparación con los pacientes con TR tratados a nivel institucional en México.

\section{Población objetivo}

El estudio busca «estimar» el costo promedio per cápita de pacientes atendidos y tratados a nivel institucional con diálisis-hemodiálisis y con TR.

\section{Horizonte del estudio}

El horizonte temporal considerado fue de un año, en el cual se incluyeron los costos promedio per cápita por paciente con diálisis-hemodiálisis y con TR, y que fueron atendidos y tratados a nivel institucional.

\section{Perspectiva del estudio}

El estudio considera la perspectiva institucional pública del sistema de salud mexicano para evaluar el costo médico directo de dos instituciones representativas (en este caso el IMSS y el ISSSTE) al atender casos en pacientes con diálisis-hemodiálisis y TR.

\section{Perspectiva del costeo}

Los costos promedios fueron evaluados desde el punto de vista de los prestadores de servicios públicos de salud. Sólo se consideraron los costos médicos directos de los pacientes (consultas médicas, laboratorios, gabinetes, cirugías, medicamentos, etcétera) que fueron hospitalizados y de los insumos y/o recursos que utilizaron en un año posterior a sus hospitalizaciones.

\section{Descripción del modelo de costeo}

Se consideraron para ambas alternativas de tratamiento (diálisis-hemodiálisis y TR) los costos médicos directos de los insumos y/o recursos que se utilizaron dentro de un horizonte temporal de un año (consultas médicas, laboratorios, gabinetes, cirugías, medicamentos, etcétera). Posteriormente se estimaron los costos promedio que generarían estos pacientes durante un seguimiento de tres y seis años.

\section{Bases de datos médicos}

A partir de los registros de atención de pacientes de ambas instituciones y de los datos reportados por el Centro Nacional de Trasplantes (CENATRA) se estimaron dos cohortes representativas de 100 
pacientes para cada alternativa de tratamiento (diálisis-hemodiálisis y TR) a fin de calcular el costo promedio de atención en el horizonte temporal establecido (inicial de un año). Para poder estimar el costo de seguimiento a tres y seis años de estos pacientes se utilizaron las probabilidades de supervivencia por año (Figura 1) en pacientes con diálisis-hemodiálisis y TR publicadas por Arredondo A y su equipo en $2011,{ }^{7}$ las cuales han sido validadas por médicos expertos en la atención de estos pacientes, tanto en el IMSS como en el ISSSTE.

Siguiendo con el caso base en el IMSS e ISSSTE, la información relativa a la frecuencia de utilización de recursos durante la hospitalización y la atención ambulatoria de los pacientes con diálisis-hemodiálisis y TR se estimó a través de la propia información de la experiencia de atención de los médicos tratantes de cada institución durante 2017.

El tipo de estudio se limitó a considerar los costos directos de la atención médica (consultas, hospitalizaciones, estudios de laboratorio y gabinete, medicamentos, etcétera) de los pacientes con diálisis-hemodiálisis y TR desde la perspectiva de un prestador de servicios específico (IMSS o ISSSTE). Con base en la experiencia de los médicos tratantes se estimó la cantidad de insumos y/o recursos que se utilizaron durante un año y su posterior seguimiento a tres y seis años (consultas médicas, hospitalizaciones, días de estancia promedio por hospitalización, complicaciones, laboratorios, gabinetes, cirugías, medicamentos, entre otros) en los pacientes con diálisis-hemodiálisis y TR (Tablas 1 a 3).

Los costos unitarios de atención se obtuvieron a partir de la propia información de cada institución; para el caso del IMSS se tomaron los costos publicados por el Diario Oficial de la Federación (DOF) de acuerdo con la Aprobación de los Costos Unitarios por Nivel de Atención Médica para $2018,{ }^{16}$ y para el ISSSTE a partir del Tabulador de Cuotas de Recuperación por la Atención Médica a pacientes no derechohabientes. ${ }^{17}$ Todos los costos se expresaron en pesos mexicanos (Tablas 4 a 6).

Cabe mencionar que el costo promedio de las complicaciones correspondió al número de eventos que, con base en la experiencia de los médicos tratantes de ambas instituciones, se presentan con mayor frecuencia en estos pacientes. Para asumir el costo institucional anual de cada complicación mencionada por los médicos tratantes se asumió el costo reportado por el IMSS en sus GRD (Grupo Relacionado por el Diagnóstico) para 2014; para llevar estos costos a precios de 2018 se utilizó el factor de actualización obtenidos de la propia información del IMSS a partir de sus costos unitarios para 2018 (Tabla 7). ${ }^{16}$

Dentro de la utilización y consumo de insumos médicos, específicamente de los medicamentos, sólo se contemplaron los utilizados para las entidades específicas, es decir, medicamentos «propios para la diálisis-hemodiálisis y TR», por lo que no se incluyeron los medicamentos utilizados en enfermedades primarias (diabetes mellitus, hipertensión arterial, glomerulonefritis, etcétera). Se tomaron, con base en la experiencia de los médicos tratantes de ambas instituciones, "esquemas tipo" de medicamentos, esto con la finalidad de poder abarcar la mayor cantidad de esquemas «propios para la diálisis-hemodiálisis y TR» que se utilizan en ambas instituciones.

El costo unitario de los medicamentos base utilizados para formar los «esquema tipo» en los pacientes con diálisis-hemodiálisis se obtuvieron de las compras realizadas por el IMSS de su portal de compras IMSS durante 2018. ${ }^{18}$

Tabla 3: Insumos utilizados por año en trasplante renal.

\begin{tabular}{|c|c|c|c|}
\hline \multirow[b]{2}{*}{ Frecuencia de insumos utilizados } & \multicolumn{3}{|c|}{ Núm. de insumos } \\
\hline & Año 1 & Año 3 & Año 6 \\
\hline Estudios pretrasplante [Rango] & $1[1-1]$ & $0[0-0]$ & $0[0-0]$ \\
\hline Cirugía del donador [Rango] & $1[1-1]$ & $0[0-0]$ & $0[0-0]$ \\
\hline Cirugía del receptor [Rango] & $1[1-1]$ & $0[0-0]$ & $0[0-0]$ \\
\hline Estudios postrasplante hospitalización [Rango] & $4.5[4-5]$ & $4[4-4]$ & $2[2-2]$ \\
\hline Estudio postrasplante ambulatorios [Rango] & $12[12-12]$ & $6[6-6]$ & $2[2-2]$ \\
\hline Consultas totales (control y seguimiento) [Rango] & 26.5 [24-29] & $3[2-4]$ & $2[1-3]$ \\
\hline Estudios de rutina mensual [Rango] & $20.5[12-29]$ & $1.5[1-2]$ & $1[1-1]$ \\
\hline
\end{tabular}


Tabla 4: Costo promedio de insumos utilizados por año en diálisis peritoneal.

\begin{tabular}{lc}
\hline Costo promedio de insumos & $\begin{array}{c}\text { Precio unitario } \\
\text { (promedio) }\end{array}$ \\
\hline $\begin{array}{l}\text { Consultas totales } \\
\text { (control y seguimiento) }\end{array}$ & $\$ 1,671.25$ \\
Estudios de laboratorio & $\$ 223.58$ \\
Estudios de gabinete & \\
(radiodiagnósticos y electrodiagnóstico) & $\$ 630.13$ \\
Insumos para la diálisis* & $\$ 14,035.00$ \\
Costo de la cirugía (catéter) & $\$ 23,571.50$ \\
\hline
\end{tabular}

*El costo promedio mensual de insumos para la diálisis está compuesto de: bolsa, material para procedimiento y cambio de línea.

Tabla 5: Costo promedio de insumos utilizados por año en hemodiálisis.

\begin{tabular}{lc}
\hline Costo promedio de insumos & Precio unitario (promedio) \\
\hline Consultas totales (control y seguimiento) & $\$ 1,671.25$ \\
Estudios de laboratorio & $\$ 223.58$ \\
Estudios de gabinete (radiodiagnósticos y & $\$ 630.13$ \\
electrodiagnóstico) & \\
Procedimiento de hemodiálisis (sesión de & $\$ 2,763.00$ \\
hemodiálisis) & $\$ 41,892.50$ \\
Cirugía para fístula arteriovenosa & $\$ 17,146.00$ \\
\hline Cirugía para implantación de catéter & \\
\hline
\end{tabular}

\section{RESULTADOS}

\section{Análisis de costos diálisis-hemodiálisis}

Los costos unitarios estimados por paciente que requirieron tratamiento con diálisis o hemodiálisis se pueden observar en las Tablas 8 y 9, respectivamente.

Se puede apreciar que para los pacientes con diálisis-hemodiálisis el costo de atención anual promedio per cápita para el año uno fue de $465,485.96$ y $783,780.44$ pesos para diálisis y hemodiálisis, respectivamente, siendo el costo por las complicaciones en el caso de la diálisis uno de los recursos de mayor costo con $254,111.27$ pesos, lo que representa $54.6 \%$ del costo total de atención. En los pacientes con hemodiálisis las sesiones representaron el insumo de mayor costo con un promedio de $397,872.00$ pesos, lo cual representa $50.8 \%$ del costo de atención. Después de las complicaciones en los pacientes con diálisis, el rubro de mayor costo fueron los insumos para la diálisis (bolsa, material para procedimiento y cambio de línea), representando $36.3 \%$ (168,420.00 pesos), y para los pacientes con hemodiálisis las complicaciones fueron el segundo rubro de atención, lo cual representa 39.0\% (305,437.08 pesos) de su costo total de atención.

En el caso del consumo de medicamentos se observó que para los pacientes que requirieron tratamiento con diálisis y hemodiálisis el costo total de los mismos representó $<1 \%$ de su costo total $(3,228.48$ y $5,237.52$ pesos para diálisis y hemodiálisis, respectivamente).

\section{Análisis de costos trasplante renal}

En la Tabla 10 se puede apreciar que, de los costos unitarios generados por los pacientes con TR, el costo de atención anual promedio per cápita para el año uno fue de 799,374.84 pesos, cuyo costo por las complicaciones al igual que la diálisis fue el que generó el mayor gasto de atención, lo cual representa $48.9 \%$ del costo total de atención con $390,548.15$ pesos, seguido por el costo de cirugía del receptor y del donador con un costo de $163,788.50$ y $81,760.00$ pesos, respectivamente; ambas cirugías representaron 20.5 y $10.2 \%$ del costo total de atención.

En el caso del consumo de medicamentos se observó un comportamiento diferente al mostrado en los pacientes bajo tratamiento con diálisis y hemodiálisis, donde el costo total de los mismos representó $5.6 \%$ de su costo total $(44,918.45$ pesos), sólo por debajo de los costos por consultas totales $(49,203.88$ pesos) y los estudios pretrasplante $(46,418.45$ pesos) que representaron 6.2 y $5.8 \%$ del costo total de atención, respectivamente.

Análisis de costos para

diálisis-hemodiálisis y trasplante

renal. Año tres y seis

Al realizar el análisis comparativo de los costos promedios generados de ambas instituciones (IMSS e

Tabla 6: Costo promedio de insumos utilizados por año en trasplante renal.

\begin{tabular}{lr}
\hline Costo promedio de insumos & $\begin{array}{c}\text { Precio unitario } \\
\text { (promedio) }\end{array}$ \\
\hline Estudios pretrasplante & $\$ 46,418.45$ \\
Cirugía del donador & $\$ 81,760.00$ \\
Cirugía del receptor & $\$ 163,788.50$ \\
Estudios postrasplante hospitalización & $\$ 494.00$ \\
Estudio postrasplante ambulatorios** & $\$ 414.33$ \\
Consultas totales (control y seguimiento) & $\$ 1,856.75$ \\
Estudios de rutina mensual** & $\$ 758.17$ \\
\hline
\end{tabular}

* Costo promedio de estudios laboratorio, radiodiagnósticos y electrodiagnóstico. Tercer nivel.

${ }^{*}$ Costo promedio de estudios laboratorio, radiodiagnósticos y electrodiagnóstico. Segundo nivel. 
Tabla 7: Costo y eventos anuales promedio de complicaciones más frecuentes.

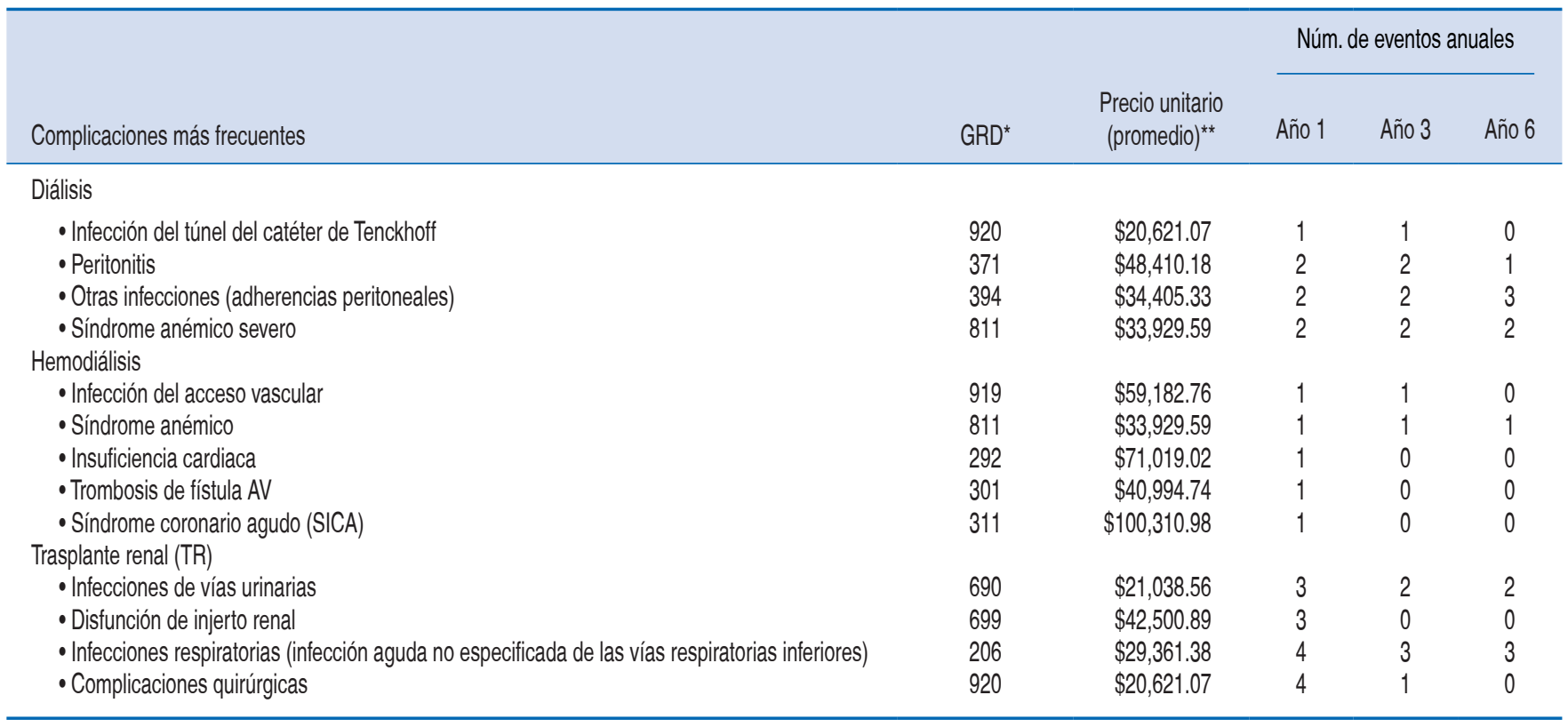

* GRD: grupos relacionados por el diagnóstico.

** Precios unitario promedio de 2018.

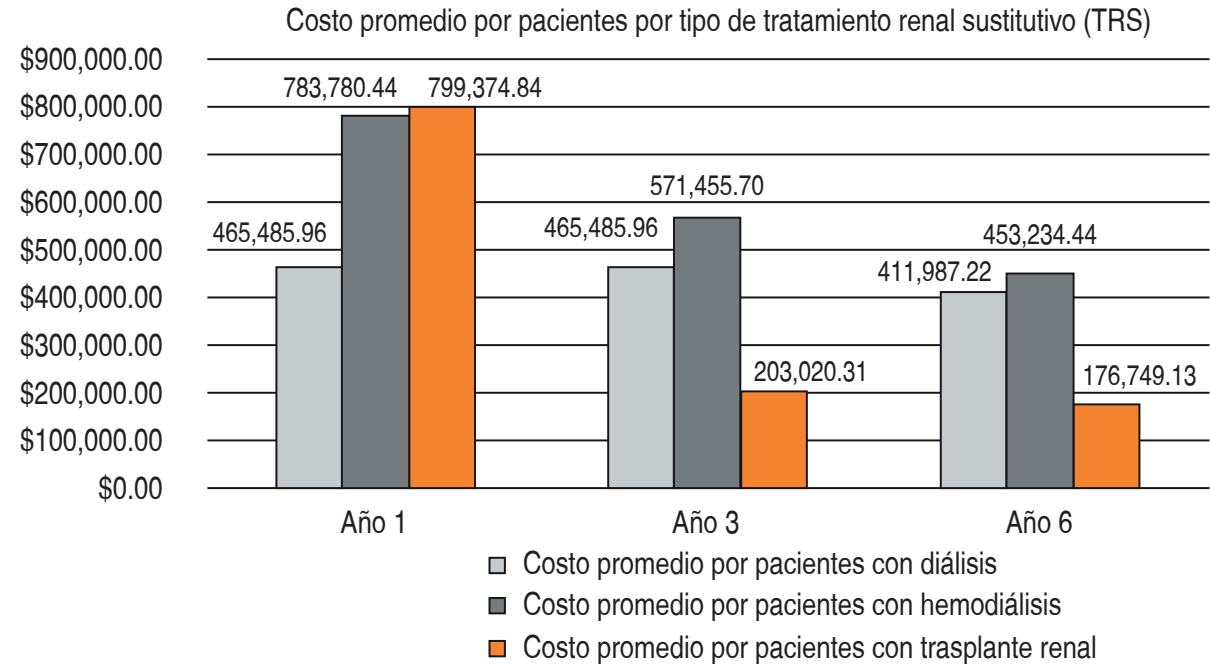

Figura 2:

Análisis de costos promedio anuales estimados en pacientes con diálisis-hemodiálisis y trasplante renal para los años 1,3 y 6 .
ISSSTE) pudimos observar que para el año uno el TR resultó ser la alternativa de tratamiento de mayor costo, presentando un costo incremental frente los pacientes de diálisis de $333,888.88$ pesos frente a los pacientes con hemodiálisis que obtuvo un costo de $\$ 15,594.40$ pesos.

Pero al realizar el seguimiento se observó una disminución en los costos de los pacientes con TR, teniendo decrementos frente a diálisis de 56 y $57 \%$, así como de 64 y $61 \%$ frente a los pacientes bajo tratamiento de hemodiálisis, para los años tres y seis, respectivamente (Figura 2).

\section{DISCUSIÓN}

En nuestro país, el número de pacientes con ERCT crece de manera significativa como consecuencia de las mejores posibilidades de diagnóstico con las que 
actualmente contamos, a esto se suma la epidemia global de diabetes mellitus tipo 2 y otras causas de ERC que tenemos en México. En nuestro país los procedimientos que se tienen para el tratamiento de la ECT son la diálisis peritoneal continua ambulatoria (DPCA), la diálisis en centro hospitalario (diálisis peritoneal intermitente y hemodiálisis) y el TR. ${ }^{19}$

Se sabe por diversas fuentes que los costos de atención en pacientes con ERC son altos, tal y como lo han descrito diversos estudios realizados en distintas partes del mundo que han tomado en cuenta las tres modalidades de la TRR (TR, diálisis peritoneal y hemodiálisis) y que han considerado las diferentes modalidades de cada uno; también se sabe que en países con ingresos económicos bajos los pacientes que ingresan a TRR, como en la diálisis, mueren o suspenden el tratamiento dentro de los tres primeros meses debido a restricciones económicas.

Otros estudios, que también han tomado en cuenta las tres modalidades de TRR (TR, diálisis peritoneal y hemodiálisis) y han considerando las diferentes submodalidades de cada uno, han demostrado el alto costo de atención de los pacientes en ERC. Se sabe que la metodología utilizada en las investigaciones es distinta, donde la mayoría coincide en que los cálculos que se realizaron fueron de forma muy general y con opiniones de expertos. Otra forma de cálculo es mediante la revisión de expedientes, que en ocasiones tiende a ser poco profunda y no incluye todos los aspectos importantes del tratamiento. Esto depende en gran medida de la calidad de los expedientes que se revisan, lo que al final puede dar como resultado información altamente cuestionable en países en vías de desarrollo. Globalmente, en la mayoría de los estudios realizados los costos fueron calculados anualmente. ${ }^{4}$

La mayoría de los estudios coinciden que, en relación con los costos resultantes de la TRR por evento, se podría concluir, en primera instancia, que como eventos aislados la diálisis y la hemodiálisis resultarían ser las terapias con un costo insignificante en relación con el costo del TR. Sin embargo, al identificar el costo de manejo anual para cada intervención, los resultados cambiaron de manera significativa, ubicando el costo de manejo anual de trasplante como el menos costoso, seguido por el costo de manejo anual con diálisis peritoneal y siendo la terapia de mayor costo anual la hemodiálisis. Si bien los costos del trasplante exceden los de la diálisis y la hemodiálisis de mantenimiento en el primer año postrasplante, éstos se reducen notablemente comparados con los de la diálisis y la hemodiálisis en los años subsiguientes, especialmente gracias al advenimiento de las drogas inmunosupresoras. ${ }^{20}$

De la misma manera, esto ha sido reportado por Arredondo y su grupo de investigación en 1998, quienes realizaron el cálculo del costo por evento, además del costo anual de las tres modalidades de TRR; después de tomar los costos de mantenimiento de las modalidades por considerar la etapa inicial del costo trasplante como una inversión, sólo se tomó el costo postrasplante anual como costo de mantenimiento. El resultado final fue que la hemodiálisis resultó ser la terapia de un mantenimiento de mayor costo en pacientes con ERC, en la que su costo de mantenimiento fue 3.2 veces mayor que

Tabla 8: Costos promedio anuales estimados en pacientes con diálisis (2018).

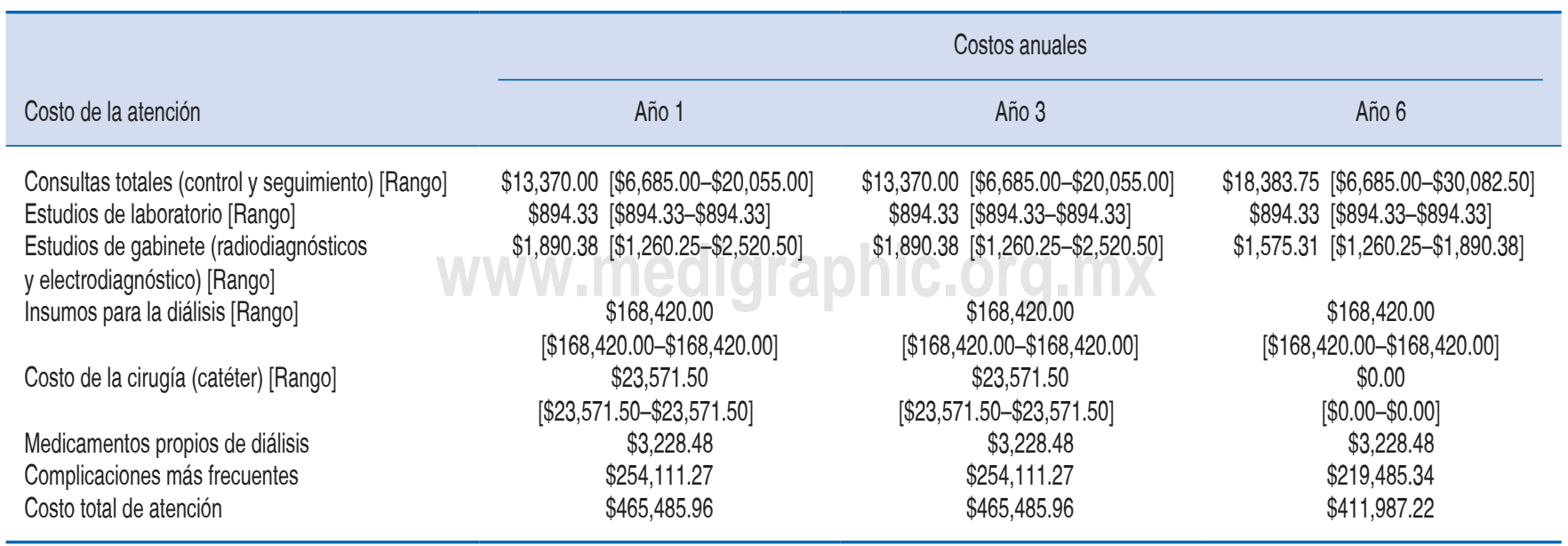


Tabla 9: Costos promedio anuales estimados en pacientes con hemodiálisis (2018).

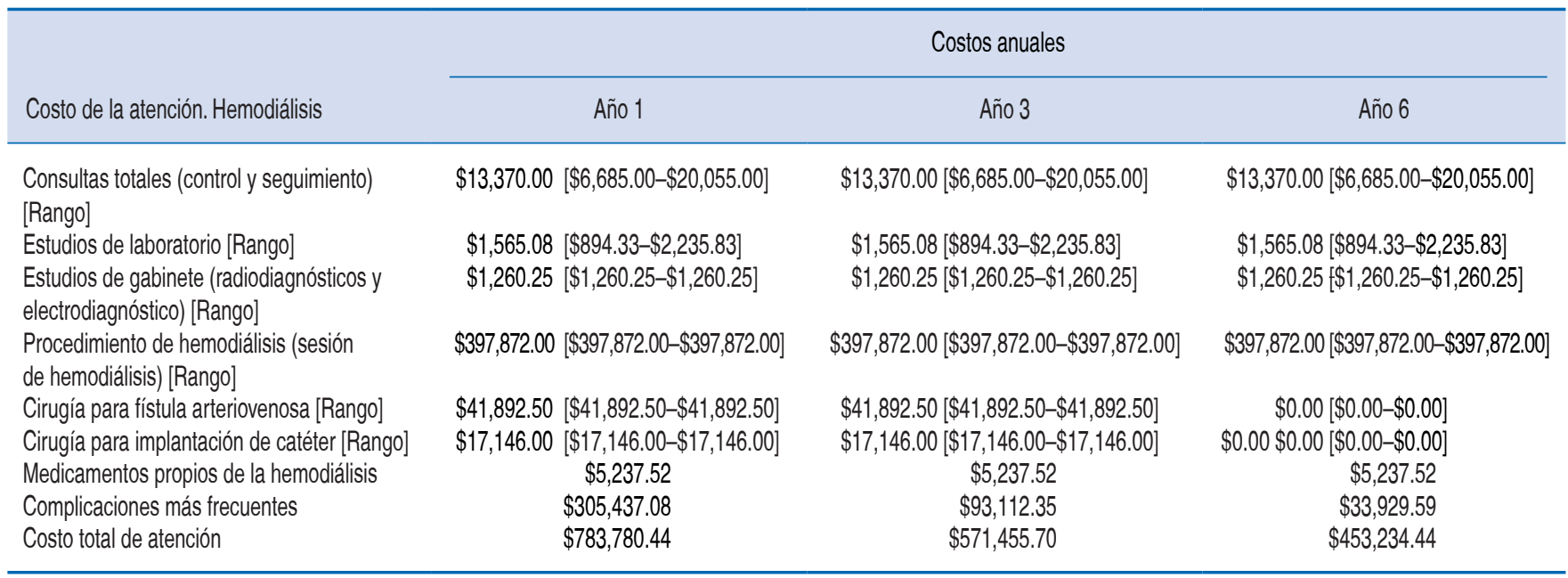

Tabla 10: Costos promedio anuales estimados en pacientes con trasplante renal (2018).

\begin{tabular}{lcrr}
\hline & \multicolumn{3}{c}{ Costos anuales } \\
\cline { 2 - 4 } Costo de la atención & Año 1 & Año 3 & Año6 \\
\hline Estudios pretrasplante [Rango] & $\$ 46,418.45[\$ 46,418.45-\$ 46,418.45]$ & $\$ 0.00[\$ 0.00-\$ 0.00]$ & $\$ 0.00[\$ 0.00-\$ 0.00]$ \\
Cirugía del donador [Rango] & $\$ 81,760.00[\$ 81,760.00-\$ 81,760.00]$ & $\$ 0.00[\$ 0.00-\$ 0.00]$ & $\$ 0.00[\$ 0.00-\$ 0.00]$ \\
Cirugía del receptor [Rango] & $\$ 163,788.50[\$ 163,788.50-\$ 163,788.50]$ & $\$ 0.00[\$ 0.00-\$ 0.00]$ & $\$ 0.00[\$ 0.00-\$ 0.00]$ \\
Estudios postrasplante hospitalización [Rango] & $\$ 2,223.00[\$ 1,976.00-\$ 2,470.00]$ & $\$ 1,976.00[\$ 1,976.00-\$ 1,976.00]$ & $\$ 988.00[\$ 988.00-\$ 988.00]$ \\
Estudio postrasplante ambulatorio [Rango] & $\$ 4,972.00[\$ 4,972.00-\$ 4,972.00]$ & $\$ 2,486.00[\$ 2,486.00-\$ 2,486.00]$ & $\$ 828.67[\$ 828.67-\$ 828.67]$ \\
Consultas totales (control y seguimiento) [Rango] & $\$ 49,203.88[\$ 44,562.00-\$ 53,845.75]$ & $\$ 5,570.25[\$ 3,713.50-\$ 7,427.00]$ & $\$ 3,713.50[\$ 1,856.75-\$ 5,570.25]$ \\
Estudios de rutina mensual & $\$ 15,542.42$ & $\$ 1,137.25$ & $\$ 758.17$ \\
Complicaciones más frecuentes & $\$ 390,548.15$ & $\$ 150,782.32$ & $\$ 130,161.25$ \\
Costo total de atención & $\$ 799,374.84$ & $\$ 203,020.31$ & $\$ 176,749.13$ \\
\end{tabular}

lo generado por los pacientes con TR y 1.7 veces mayor que el costo anual de mantenimiento de pacientes en diálisis. ${ }^{19}$

Los resultados obtenidos del costo anual estimado promedio de la atención del paciente con ERC con las tres modalidades de TRR (TR, diálisis peritoneal y hemodiálisis) en nuestro estudio coinciden con lo publicado a nivel internacional, en donde se concuerda que en el primer año el TR es la alternativa de mayor costo considerando los costos mismos del trasplante, pero al realizar el seguimiento se puede observar que al tercer año de mantenimiento con las tres modalidades de TRR el costo de atención por hemodiálisis resultó ser la alternativa de mayor costo, la cual es 2.8 veces mayor que los pacientes en seguimiento con trasplante y 1.2 veces mayor que los pacientes en diálisis peritoneal.

\section{CONCLUSIONES}

De los resultados obtenidos respecto al costo anual del manejo del paciente con ERC, sin tomar en cuenta los criterios de efectividad, el TR resultó ser la alternativa de menor costo para el manejo de estos pacientes, seguido por la diálisis; caso contrario a la hemodiálisis, que fue la terapia más costosa. Se puede concluir sólo desde una perspectiva económica que el TR efectivamente debería ser la intervención de mayor promoción y aplicación en cuanto a las tres intervenciones del estudio.

El trasplante preventivo preingreso a diálisis es una opción atractiva tanto para los pacientes como para los pagadores $y / 0$ instituciones de salud, ya que reduce los costos y mejora la supervivencia del injerto. EI TR apropiadamente indicado es el tratamiento de elección 
para los pacientes con ERC, debido a sus menores costos y a que ofrece una mejor evolución de la enfermedad. Sin embargo, existen sustanciales desigualdades en el acceso al trasplante en el mundo, pues tan sólo en México, de acuerdo con cifras de CENATRA, para 2017, del total de pacientes que requerían un trasplante sólo $18.7 \%$ recibió un TR $(3,150)$ y $81.3 \%$ $(13,634)$ de los pacientes se encontraban en lista de espera de un riñón, lo que conlleva a resaltar que los resultados mostrados como en este estudio dan argumentos para ampliar el programa de trasplantes y a su vez sugerir la ampliación de programas de procuración de órganos provenientes del donante fallecido.

\section{AGRADECIMIENTOS}

Agradecemos a Luis Camacho Cordero el apoyo editorial financiado por Pfizer.

\section{REFERENCIAS}

1. Tratamiento sustitutivo de la función renal. Diálisis y hemodiálisis en la insuficiencia renal crónica. México: Secretaría de Salud; 2014.

2. Ávila-Saldivar MN, Conchillos-Olivares G, Rojas-Báez IC, Ordoñez-Cruz AE, Ramírez-Flores HJ. Enfermedad renal crónica: causa y prevalencia en la población del Hospital General La Perla. Med Int Mex. 2013; 29: 473-478.

3. Méndez-Durán A, Méndez-Bueno JF, Tapia-Yáñez T, Muñoz Montes A, Aguilar-Sánchez L. Epidemiología de la insuficiencia renal crónica en México. Dial Traspl. 2010; 31 (1): 7-11.

4. López CM, Rojas RM, Tirado GL, Durán AL, Pacheco DR, Venado EA et al. Enfermedad renal crónica y su atención mediante tratamiento sustitutivo en México. México: Facultad de Medicina, Universidad Nacional Autónoma de México; 2009.

5. Franco-Marina F, Tirado-Gómez L, Venado-Estrada A, MorenoLópez J, Pacheco-Domínguez R, Durán-Arenas L, LópezCervantes M. Una estimación indirecta de las desigualdades actuales y futuras en la frecuencia de la enfermedad renal crónica terminal en México. Salud Pública Mex. 2011; 53 Supl 4: S506-S515.

6. Cortés-Sanabria L, Ayala-Cortés RA, Calderón-García CE, Silva-Ocegueda A. Retos y perspectivas de la enfermedad renal crónica en México: a propósito del día mundial del riñón, 2017. Rev Sal Jal. 2017; 4 (1): 6-9.

7. Arredondo A. Costos de intervenciones para enfermedades crónicas en México. Informe Técnico. INSP. 2011; 2012: 37-48.

8. Centro Nacional de Trasplantes (CENATRA). Reporte Anual 2017 de Donación y Trasplantes en México. Sistema Informático del Registro Nacional de Trasplantes, corte 31 de Diciembre de 2017.
9. Centro Nacional de Trasplantes (CENATRA). Estado Actual de Receptores, Donación y Trasplantes en México. Primer. Semestre 2018. Sistema Informático del Registro Nacional de Trasplantes, corte al 03 de julio de 2018.

10. Wolfe RA, Ashby VB, Milford EL, Ojo AO, Ettenger RE, Agodoa LY et al. Comparison of mortality in all patients on dialysis, patients on dialysis awaiting transplantation and recipients of a first cadaveric transplant. N Engl J Med. 1999; 341 (23): 1725-1730.

11. Azar SA, Nakhjavani MR, Tarzamni M, Faragi A, Bahloli A, Badroghli N. Is living kidney donation really safe? Transplant Proc. 2007; 39: 822-823.

12. Informe al Ejecutivo Federal y al Congreso de la Unión sobre la situación financiera y los riesgos del Instituto Mexicano del Seguro Social 2012-2013 [Internet]. Consejo Técnico IMSS, 2013. Disponible en: http://www.imss. gob.mx/instituto/informes/ Pages/index.aspx.

13. Administración del Censo de pacientes con Insuficiencia Renal Crónica (CIRC). México: Instituto Mexicano del Seguros Social; 2014.

14. Arredondo A, Rangel R, De Icaza E. Costos de intervenciones para pacientes con insuficiencia renal crónica. Rev Saude Publica. 1998; 32 (3): 255-261.

15. Arredondo A, Rangel R, De Icaza E. Costo-efectividad de intervenciones para insuficiencia renal crónica terminal. Rev Saude Publica. 1998; 32 (6): 556-565.

16. Diario Oficial de la Federación (DOF), relativo a la Aprobación de los Costos Unitarios por Nivel de Atención Médica actualizados al año 2018 [Jueves 28 de diciembre de 2017].

17. Tabulador de Cuotas de Recuperación por la Atención médica a pacientes no Derechohabientes del ISSSTE. 2018. Prosecretaria de la Junta Directiva. Secretaría General. Instituto de Seguridad y Servicios Sociales de los Trabajadores del Estado (ISSSTE).

18. Compras.imss.gob.mx [Internet]. México: Instituto Mexicano del Seguro Social; 2018 [última visita 02 de noviembre del 2018]. Disponible en: http://compras.imss.gob.mx/?P=imsscompro.

19. Arredondo A, Rangel R, de Icaza E. Costo-efectividad de intervenciones para insuficiencia renal crónica terminal. Rev Saude Publica. 1998; 32 (6): 556-565.

20. García G, Harden P, Chapman J. World Kidney Day Steering Committee 2012. El papel global del trasplante renal. Nefrologia. 2012; 32 (1): 1-6.
Correspondencia:

Aczel Sánchez-Cedillo

Centro Médico Nacional «20 de Noviembre», Laboratorio de Trasplantes.

Av. San Lorenzo Núm. 501, Edificio «D», 2o. piso, Col. Del Valle, 03100 , Alcaldía Benito Juárez, Ciudad de México.

Tel. (55) 52005003, ext. 50171/14384

E-mail: aczel@ciencias.unam.mx 